\title{
Performance Comparisons of Contour-based Corner Detectors
}

\author{
Mohammad Awrangjeb*t, Guojun $\mathrm{Lu}^{\ddagger}$ and Clive S. Fraser ${ }^{\dagger}$
}

\begin{abstract}
Corner detectors have many applications in computer vision and image identification and retrieval. Contour-based corner detectors directly or indirectly estimate a significance measure (e.g., curvature) on the points of a planar curve and select the curvature extrema points as corners. While an extensive number of contour-based corner detectors have been proposed over the last four decades, there is no comparative study of recently proposed detectors. This paper is an attempt to fill this gap. The general framework of contour-based corner detection is presented and two major issues - curve smoothing and curvature estimation, which have major impacts on the corner detection performance, are discussed. A number of promising detectors are compared using both automatic and manual evaluation systems on two large data sets. It is observed that while the detectors using indirect curvature estimation techniques are more robust, the detectors using direct curvature estimation techniques are faster.
\end{abstract}

\section{Index Terms}

corner detection, performance study, CPDA, Fast-CPDA.

EDICS: ARS-RBS Region, Boundary, and Shape Analysis, ARS-IVA Image \& Video Mid Level Analysis.

\section{INTRODUCTION}

The terms 'dominant point', 'critical point' and 'corner' are taken as equivalent in the literature to indicate detected corners on a planar curve (open or close contour) [48]. Although the notion

(C) Copyright (c) 2012 IEEE. Personal use of this material is permitted. However, permission to use this material for any other purposes must be obtained from the IEEE by sending a request to pubs-permissions@ieee.org.

*corresponding author.

${ }^{\dagger}$ Cooperative Research Centre for Spatial Information, Department of Infrastructure Engineering, University of Melbourne, Vic 3010, Australia, Phone: +61 38344 9182, Fax: +61 39349 5185, E-mail: mawr@unimelb.edu.au (M. Awrangjeb) and c.fraser@unimelb.edu.au (C. S. Fraser).

${ }^{\ddagger}$ Gippsland School of IT, Monash University, Churchill Vic 3842, Australia, Phone: +61 39902 6857, Fax: +61 35122 7137, E-mail: Guojun.Lu@infotech.monash.edu.au. 
of a 'corner' seems to be intuitively clear, no generally accepted mathematical definition exists for digital curves [16]. In fact, different approaches give different but conceptually related computational definitions of a visual phenomenon. For example, Guru et al. [17] defined a corner as the intersection of two adjacent relatively straight curve-segments. Thus, a corner point is found at a location where the direction of the curve changes significantly and abruptly.

Although the terms 'interest-point' and 'corner' are sometimes used equivalently in the literature, corners are a special subclass of interest-points. Interest-points include not only corners, but also T-junctions and blobs, as well as locations of significant texture variation [23]. While contour-based corner detectors first perform edge detection, and then analyse the edges to find the locations of rapid changes in direction (ie, corners), interest-point detectors no longer require an explicit edge detection step and they search for high levels of curvature directly in the image gradient. Consequently, interestpoint detectors may detect image points that are not corners in the traditional sense, a small bright spot on a dark background, for instance. A comprehensive review and performance comparison of interest-point detectors can be found in [24], [41].

Recently, a number of promising contour-based detectors have been proposed. However, a comparative study to assess each of them on a common platform has yet to be reported. This paper is an attempt to fill this gap. Note that the earlier comparative work by Mokhtarian and Mohanna [26] is almost six years old. Moreover, they used a small data set and their evaluation system was manual and consisted of flawed evaluation metrics [5]. In this paper, there are two major contributions as follows. Firstly, a comprehensive analysis of contour-based detectors under a general framework of contour-based corner detection is presented. Secondly, a thorough comparative study of eleven promising detectors, performed using both automatic [6] and manual [26] evaluation systems on two large data sets, is reported. A ranking of detectors is then made on the basis of results obtained.

The rest of the paper is organized as follows. Sections II and III present the general framework of contour-based corner detection and discuss two major issues, namely curve smoothing and curvature estimation. Both have major impacts on corner detection performance. Section IV initially summarizes previously reported results, and then offers comparison of the eleven promising detectors. Finally, concluding remarks are made in Section V.

\section{General Framework of Contour-based Corner Detection}

As shown in Fig. 1, common contour-based corner detection techniques comprise of five steps: edge extraction and selection, curve smoothing, curvature estimation, finding corners and coarse-tofine corner tracking. The 'corner tracking' step indicated in Fig. 1 is optional and often not used by many detectors. The 'curve smoothing' and 'curvature estimation' steps are sometimes integrated, as indicated in the figure, for example, for detectors estimating indirect curvature via an indirect 


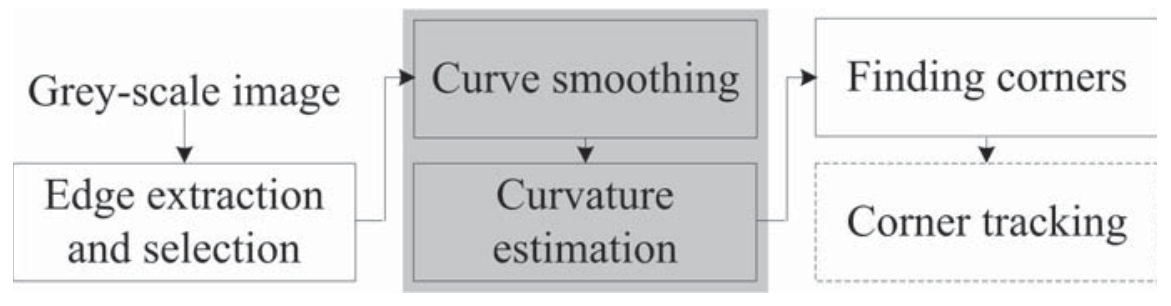

Fig. 1. Five steps for contour-based corner detection.

smoothing technique.

- Edge extraction and selection: Given a grey-scale image, a corner detector first extracts edges using an edge detector. Most of the corner detectors in the literature do not detail this step since edge extraction is a separate algorithm and commonly used by all corner detectors. However, Awrangjeb and Lu [5] have shown that extraction of strong edges and elimination of short and weak edges, which may be large in number, can increase the performance of a corner detector.

- Curve smoothing: Evaluating the slope and curvature of a discrete curve is not simple, since the position (coordinates) of a curve-point is quantized. Moreover, there may be noise and local variation on the curve. Therefore, a degree of smoothing should be carried out before or during the curvature calculation in order to make the curvature extrema points more distinguishable from other curve-points.

There are two kinds of smoothing: direct and indirect. A direct smoothing, e.g. the Gaussian smoothing [27], [32], removes noise and changes the curve-point locations, while an indirect smoothing, e.g. the region of support (RoS) [36] or the chord-length [33], does not change the curve-point locations. As the size of the RoS or chord is increased, the curvature estimation technique can overlook fine detail of the curve without changing the curve-point locations to any practical extent. Both kinds of smoothing have a similar effect in the sense that using a low value for the smoothing parameter may not significantly reduce the effect of noise and may detect many spurious corners, while using a large value for the smoothing parameter may overlook important detail in the curve.

Since detectors using indirect smoothing do not physically remove noise from the curve, they may detect spurious corners and may not yield satisfactory corner localization. Successful corner detection requires some direct curve smoothing to be performed beforehand [2]. Rosenfeld and Johnston [36] and Rosenfeld and Weszka [37] reported the importance of direct curve smoothing (Gaussian smoothing with scale $\sigma$ ) in their earlier work, though they did not apply smoothing. Awrangjeb and Lu [6] applied a small Gaussian smoothing-scale (direct smoothing) before the 
estimation of curvature using chords (indirect smoothing). In addition to using a fixed Gaussian smoothing, He and Yung [18] used a dynamic (adaptive) RoS to calculate the variable curvaturethreshold.

- Curvature estimation: A corner detector estimates a given significance measure, i.e. curvature, for each point of the smoothed curve. The estimated curvature is sometimes referred as "corner strength' or 'cornerity' [4].

There are two types of curvature estimation techniques in the literature: direct and indirect. In direct curvature estimation, an algebraic or geometric estimation such as cosine, local curvature or tangential deflection [27], [37], [32], [30] at each curve-point is used as the significance measure. Such algebraic or geometric estimations are measured at each point with its neighbouring points. For example, Mokhtarian and Suomela [27] estimated the Euclidean curvature (local curvature) at each point considering two neighbouring points on each side.

In contrast, in indirect curvature estimation, indirect measures are used as significance measures. For example, Masood and Sarfraz [21] counted the number of curve-points lying inside three rectangles moving along the curve. Ramer [33] used the perpendicular distances from the chord connecting the two end-points of the curve (segment) to curve-points. Alternative indirect measures of the significance are area [49] and distance [46] functions, eigenvalues [40] and eigenvectors [44] of the covariance matrix, and the determinant of the gradient correlation matrix [47]. Since direct curvature estimation techniques consider less neighbouring points on each side of a point, they are more sensitive to noise and local variation of the curve than their indirect counterparts [6] (see detail in Section III-B).

- Finding corners: A corner detector gathers the curvature maxima points on curves in the candidate corner set. Corner detectors can be categorized into three groups based on how they select the final corners from the candidate set. In the first group, there are algorithms [27] which apply thresholds to the candidate corners in order to select strong corners and remove weak (also known as 'round' [27]) and false corners [18]. Candidate corners that survive after the application of the thresholds are then deemed to be the final corners. Mokhtarian and Suomela [27] applied a single threshold to decide the final corner set. He and Yung [18] and Awrangjeb and Lu [6] used a curvature-threshold to remove weak corners and an angle-threshold to remove false corners. Algorithms, for example, Pedrosa and Barcelos [30], in the second group directly decide curvature extrema points as corners without considering any curvature thresholds.

The third group comprises algorithms [32] that obtain a piecewise linear polygonal approximation of the shape (closed contour of an object) subject to some constraints on the goodness of fit. The actual or extrapolated intersections of the adjacent line segments in the approximated polygon are then detected as dominant points [33]. Masood [22] applied a recursive optimization algorithm 
to delete one point at a time (with minimum approximation error) from the candidate set until the remaining points achieved their optimal positions. The optimal position for a candidate point was searched within its two neighbouring candidate points where the approximation error was minimized. Carmona-Poyato [11] suppressed the collinear candidate points if the distance from a candidate point to the straight line joining two of its neighbouring candidate points was below a predefined threshold. Parvez and Mahmoud [29] improved this detector by applying an adaptive optimization algorithm for collinear candidate point suppression. Pinheiro and Ghanbari [32] first selected the candidate corners on a high smoothing-scale $(\sigma=1024)$ and then added new corners in the lower scales until a desired level of approximation of the input shape was achieved.

- Corner tracking: If corners are detected using high Gaussian smoothing-scales $(\sigma)$, their localization is generally not good. A coarse-to-fine corner tracking is carried out for the detected corners to improve their localization. For example, Mokhtarian and Suomela [27] detected corners at $\sigma=4$ and tracked them through $\sigma=3$ and 2 to $\sigma=1$. At each scale (say, $\sigma=2$ ) the point of maximum curvature around a corner (which was detected at the immediate higher scale, i.e. $\sigma=3$ ) is selected as the next tracked position for that corner. The tracked position at $\sigma=1$ is the final location for that corner. A similar approach has been adopted by Pinheiro and Ghanbari [32].

Note that the corner tracking step uses no threshold and changes the corner positions only, not the number of corners. This corner tracking step is optional in many detectors [18], [6] that use low $\sigma$ values for smoothing.

\section{MAJor IsSUes of CORNer Detection}

Curve smoothing and curvature estimation are the most critical steps in corner detection. In order to detect all true corners at their correct locations, and avoid detection of any false or weak corners on a curve, it is very important that the curve be first smoothed with an appropriate smoothing-scale. However, selection of an appropriate smoothing-scale for a given curve is not an easy task.

On the other hand, curve-point locations are quantized and there may be noise and local variations on the curve. In addition, geometric transformations or signal processing may affect the edge detection performance. As a result, it is very hard to consistently estimate curvature at a curve-point under different conditions.

In this section, additional aspects of the selection of smoothing-scale and curvature estimation techniques are discussed. 


\section{A. Smoothing-scale Selection}

A smoothing parameter, which indicates the amount of direct or indirect smoothness for curvature estimation, is employed to control the overall corner detection performance. There are three smoothing parameters referred to in the literature: Gaussian smoothing-scale $\sigma$ [26], chord-length $L$ [6] and RoS $k$ points on both sides of a curve-point [37], [30]. These will be referred to as smoothing-scales in this paper. While the first one causes a direct smoothing of the curve, the latter two are applied for indirect smoothing. A corner detector may use one [27] or more [6] of these three smoothing parameters.

In direct smoothing using $\sigma$ [27], each curve-point $P$ is convolved using a Gaussian function whose window size is determined by $\sigma$. While a large $\sigma$ ensures a high degree of smoothness by involving a high number of neighbouring points on both sides of $P$ in the convolution operation, a small $\sigma$ offers a low degree of smoothness. In indirect smoothing using $L$ [6], the parameter value indicates how many neighbouring points on both sides of $P$ are considered during curvature estimation. In indirect smoothing using $k$ [37], the parameter value indicates how many neighbouring points on both sides of $P$ are considered during the search for local curvature maxima. Sometimes $k$ is also used for the same purpose as $L$. For example, Zhang et al. [47] estimates curvature using a $1 \times 1$ neighbourhood around each curve-point.

Direct smoothing is mainly performed before curvature calculation [27], [46], [6], [26], [32] and indirect smoothing is typically used during curvature estimation [6]. In addition to a direct smoothing before curvature estimation, many detectors [25], [18], [37] also employ direct [25] or indirect smoothing [18], [37] after curvature calculation. Mokhtarian and Mohanna [25] applied direct smoothing on the estimated curvature function and He and Yung [18] and Rosenfeld and Weszka [37] applied an indirect smoothing $(\operatorname{RoS} k)$ to determine local curvature maxima points.

Detectors using a single smoothing-scale [1] suffer from the problem of selecting an appropriate value for $\sigma, L$ or $k$ for a given curve. The reason is that there may be different types and sizes of corners on a single curve. Moreover, the human visual system (HVS) shows two notable characteristics [16]. Firstly, the human visual perception treats images on several scales simultaneously. Secondly, when the signal-to-noise ratio decreases in an image, the HVS automatically increases the scales at which it characterizes the image. In addition, the detected corners using a single smoothing-scale may also be ill-positioned [49]. Therefore, it is preferable to detect corners using a multi-scale analysis.

Multi-scale corner detectors using a full range of smoothing-scales [34], [32] are computationally too expensive. In addition, the combination of corners detected in different scales presents a further problem. In order to avoid such problems, recently developed contour-based detectors detect corners on each curve using either a fixed or one of three medium smoothing-scales depending on the curve- 
length. The detected corners are then tracked to improve their localization [27], [25].

As another alternative solution to the problems of using a full range of scales, Rosin [38] represented curves at their natural (significant) scales. This representation describes each part of a curve at its significant scale (for which certain criteria are met). As a result, each curve is represented using many significant scales, instead of using the full range of scales. Gao et al. [15] proposed a multi-scale corner detector based on local natural scale. However, local approaches are computationally more expensive and coarse scale features cannot be effectively detected using the local curve nature [38]. Beau and Singer [9] suggested only coarse feature detection in the reduced resolution curve to reduce the computational complexity, because large scale features are more stable in image transformations and can be better used in different applications.

There are also adaptive smoothing (also known as non-linear filtering or anisotropic diffusion of the signal) techniques [35], [30] for corner detection. The idea behind adaptive smoothing is to apply a versatile operator that can adapt to the local topography of the signal. Though the adaptive technique improves the localization of the detected corners without following a tracking step, such an iterative adaptation of the smoothing-scale is computationally demanding. Pedrosa and Barcelos [30] applied the anisotropic diffusion directly to the curvature function. Therefore, the diffusion parameter $\rho$ directly change the curvature function (i.e., direct smoothing). Like other smoothing parameters discussed above, while a large value of $\rho$ indicates a high degree of smoothing, a small value of $\rho$ indicates a low degree of smoothing.

Due to the fact that detectors using either a full rage of multi-scales [34], significant-scales [15] or adaptive-scales [35], [30] are computationally demanding, the recent trend has been to detect corners using either a single-scale [18], [47] or a small range of multi-scales (e.g., two [46], [7] or three [6], [45] smoothing-scales).

\section{B. Curvature Estimation}

Curve-point locations are quantized and may be affected by noise introduced during an image processing operation and by the edge extractor. In addition, a curve may contain different sized corners which may require different amounts of smoothing. However, choosing or calculating an appropriate value for $\sigma, L, k$ or $\rho$ is very difficult for a given curve. As a result, there exists no strict mathematical definition of curvature for a discrete curve and curvature is only approximated [49].

As discussed in Section II, there are two main types of curvature estimation techniques - direct and indirect [49]. In this section, their technical details are presented with examples in order to show why the indirect curvature estimation techniques are more stable than the direct curvature estimation techniques. 


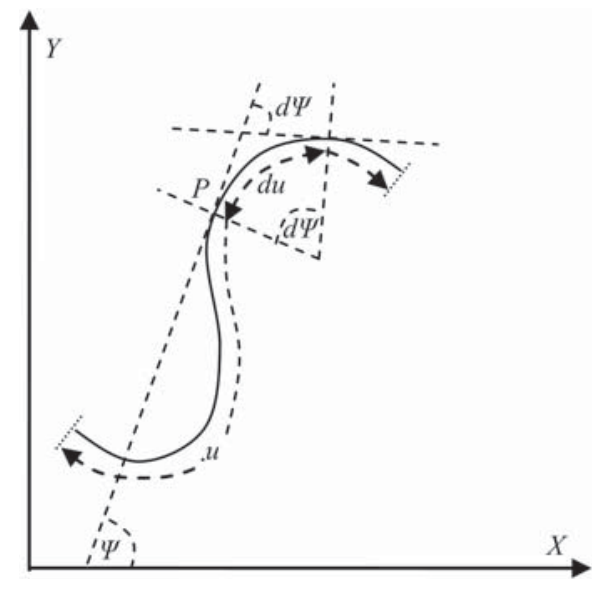

Fig. 2. Intrinsic definition of curvature.

1) Direct curvature estimation: The early dominant point detectors estimated angle as a significant measure (curvature measures such as cosine angle or tangential deflection) at each point $P$ of the curve by considering a fixed $\operatorname{RoS} k$. However, many modern dominant point detectors calculate a variable $k$ on both sides of $P$ with the expense of an additional computational cost. Depending upon the above RoS determination criterion, dominant point detection algorithms were divided into two groups. Firstly, algorithms with non-adaptive RoS keep $k$ fixed for all curves and points [36], [37], [30]. Secondly, algorithms with adaptive RoS calculate RoS for each point using the local nature of the curve [39], [20].

Many recent corner detectors [34], [27], [32] use the Euclidean curvature. The Euclidean curvature $\mathcal{C}$ at a point $P$ is defined as the instantaneous rate of change of $\psi$, that is the angle subtended by the tangent at $P$ with the $x$-axis, with respect to the arc-length $u$ [34] (see Fig. 2):

$$
\mathcal{C}(u)=\frac{d \psi}{d u} .
$$

This curvature estimation technique considers a very small neighbourhood $(2 \times 2)$ on both sides of $P$ to evaluate the curvature at $P$ [6]. Consequently, the estimated curvature using the above definition is very sensitive to the local variation and noise along the curve. In a region with a high local variation, $\psi$ changes significantly from point to point within a short curve segment. As depicted in Fig. 3, in a small but highly variable curve-region, the derivatives of the curve point-locations may lead to a high curvature estimation. As a result, if such local variation and noise are not initially smoothed away using a high smoothing-scale, detectors utilizing the direct curvature estimation may detect many weak and false corners. However, smoothing has its own problems as discussed in Section III-A.

The Euclidean curvature in (1) can also be expressed as the reciprocal of the radius of an osculating 


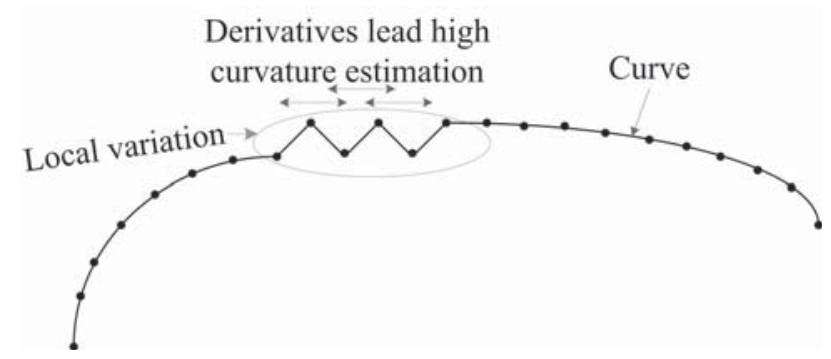

Fig. 3. Derivative-based (direct) curvature estimation within a local variation of a curve.

circle along the curve. There is a unique circle that passes through each point $P$ of the curve and most closely approximates the curve near $P$. Nguyen and Debled-Rennesson [28] considered the circle passing through $P, P_{l}$ and $P_{r}$, where $P_{l}$ and $P_{r}$ are the left and right end points of the RoS $k$ for $P$. Therefore, this curvature estimation technique considers a large neighbourhood and is less sensitive to noise, but depends on how insensitive $k$ is to noise.

Detectors using cosine angle or tangential deflection [36] were found to be more sensitive to noise and less robust than those using the Euclidean curvature [6].

Detectors using direct curvature estimation techniques may use no smoothing [37], direct smoothing only [27], [46], [13] or both direct and indirect smoothing [18], [30].

2) Indirect curvature estimation: Since direct curvature estimation techniques are sensitive to noise and local variation of the curve, there are a number of detectors which use different curvature estimation techniques. For example, the detector in [13] used the distance from a curve-point $P$ to a chord of length $L$ as a significance measure on that point. Teh and Chin [39] used the ratio of the distance between $P$ and the chord connecting the two end points $P_{l}$ and $P_{r}$ of a dynamic RoS to the length of the chord. However, such measurements were noted as sensitive to noise [10]. Wu [43] and Carmona-Poyato et al. [10] used the average of the bending values within the adaptive RoS. Wu [43] determined the bending value as the maximum of the distances in $x$ and $y$ directions from $P$ to $P_{l}$ and $P_{r}$, whereas Carmona-Poyato et al. [10] estimated the average of two vectors $\overrightarrow{P P_{l}}$ and $\overrightarrow{P P_{r}}$.

The CPDA detector [6] followed a distance accumulation technique using three chords as shown in Fig. 4. Since it uses a large neighbourhood, it is less sensitive to noise and local variations on the curve [6]. Particularly, chord 1 (short) accumulates (sums) both positive and negative distances and chord 2 (long) accumulates distances which are almost the same for all points inside and outside this region. So the curvature product from the accumulated distances using three chords of different lengths is almost the same for all the points in and out of the local variation region. As a result, the CPDA detector detects corners with a higher average repeatability. 


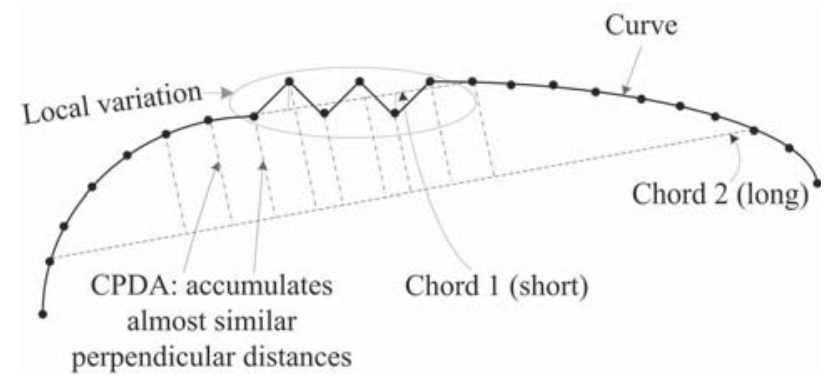

Fig. 4. CPDA (indirect) curvature estimation within a local variation of a curve.

Many detectors involving polygonal approximation [22], [11], [29] use the Freeman chain code [14] to select the candidate corner set. Marji and Siy [20] used the number of times that a curve point was selected as an end point while computing the dynamic RoSs for other curve-points.

Detectors using indirect curvature estimation techniques may use direct smoothing only [46], indirect smoothing only [13] or both [6]. They usually integrate indirect smoothing technique with curvature estimation [13], [6]. Zhang et al. [46] estimated indirect curvature without integrating or using an indirect smoothing technique.

\section{Performance Study}

Many contour-based detectors [27], [34] do not use any evaluation metrics. Instead they show the detected corners on a small number of artificial and real images. In this section, experimental results from selected recent papers [26], [45], [18], [5], [6], [46], [7], [47] are first summarized. An empirical study on a number of popular corner detectors is then presented using both the automatic [5] and manual [26] performance evaluation systems on two large data sets.

Note that the aim of the reported performance study has been to estimate the accuracy and robustness of promising contour-based detectors when they are applied to real-world images. Therefore, evaluation systems and indices [22], [11], [12] used for evaluating polygonal approximation algorithms are not included in this study.

\section{A. Summary of Existing Evaluations}

Table I compares the evaluation systems and reported test results for selected contour-based detectors. It is observed that many of the existing evaluation systems are manual (M), meaning they involved human judgement. Awrangjeb and Lu [5], [6] first introduced the automatic evaluation system (A) and included geometric sheared and lossy JPEG compressed images into the test data set. Many authors [26], [45], [18] have used a small set of images and He \& Yung [18] did not 
use any geometrically transformed or signal processed images. This makes their evaluation outcomes less reliable. As compared to five most promising detectors, Awrangjeb and Lu [6] showed that their CPDA detector offered the optimal effectiveness in terms of both average repeatability and localization error.

Though the GCM detector [47] outperformed the CPDA detector [6] in the study by Zhang et al. [47], the opposite was found in both of the manual and automatic performance studies presented in the following subsections. The reasons were possibly, unlike Zhang et al. [47], the CPDA detector [6] used standard parameters recommended by the respective authors for each detector and involved JPEG compressed and sheared images in the test data set.

Since, the evaluation results provided in Table I were carried out on different platforms (different evaluation systems using different data sets and metrics), an automatic evaluation of eleven popular corner detectors has been carried out using - average repeatability and localization error for effectiveness evaluation [6] and running time for efficiency evaluation. In addition, in order to show the accuracy of the detected corners with respect to results achieved via human perception, the manual evaluation system in [26] has been adopted and the detectors have been evaluated in terms of accuracy and localization error.

\section{B. Current Evaluation}

1) Performance indices: In the automatic system [6], corners detected in the original images by a detector are considered as reference corners, and corners detected by the same detector in the test images, which were signal processed and geometrically transformed, are taken as test corners. Then, reference and test corners are compared to evaluate the performance of that detector.

Two evaluation metrics from Awrangjeb and Lu [6] were used for robustness tests of detected corners. Repeatability $R$ indicates how stable the detected corners are under different geometric transformations and signal processing operations. Average repeatability is defined as:

$$
R_{\text {avg }}=\frac{N_{r}}{2}\left(\frac{1}{N_{0}}+\frac{1}{N_{t}}\right) .
$$

where $N_{0}$ and $N_{t}$ are the number of corners in the original and test images, respectively and $N_{r}$ is the number of repeated corners between them. Localization error shows how accurately a detected corner is localized by the detector, and it is measured using the root-mean-square-error (RMSE) of the detected corners:

$$
L_{e}=\sqrt{\frac{1}{N_{r}} \sum_{i=1}^{N_{r}}\left[\left(x_{t i}-x_{o i}\right)^{2}+\left(y_{t i}-y_{o i}\right)^{2}\right]} .
$$

Here $\left(x_{o i}, y_{o i}\right)$ and $\left(x_{t i}, y_{t i}\right)$ are the original and test positions of the $i$-th repeated corner. An RMSE value of a maximum 3 pixels was allowed to find a corner correspondence or repetition. 


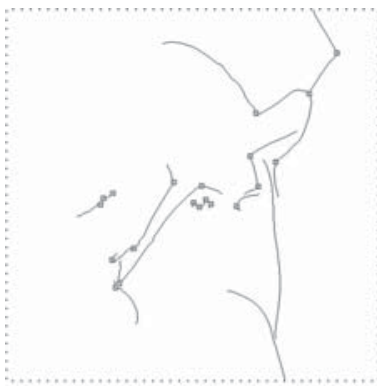

(a)

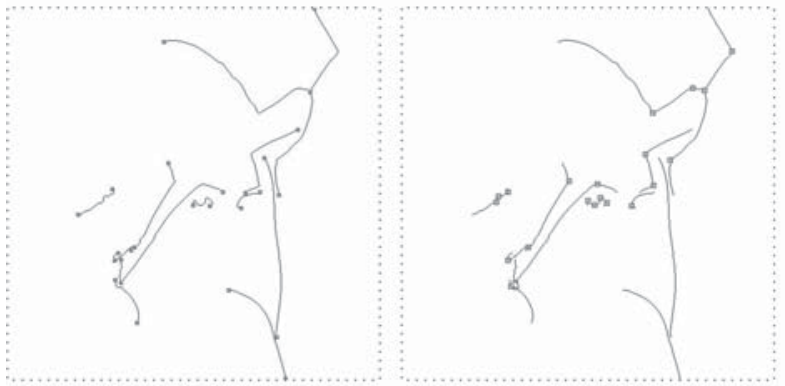

(e)

(f)

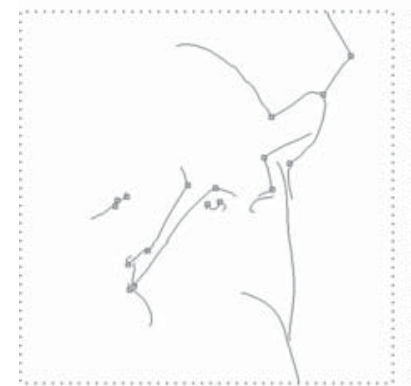

(i)

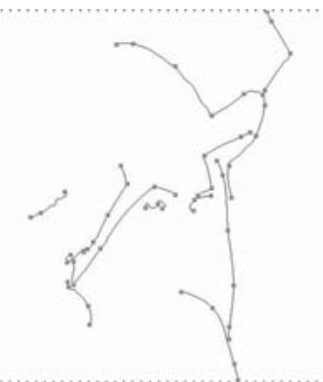

(b)

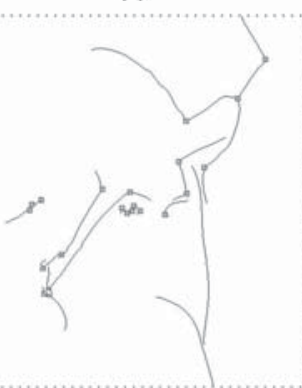

(j)

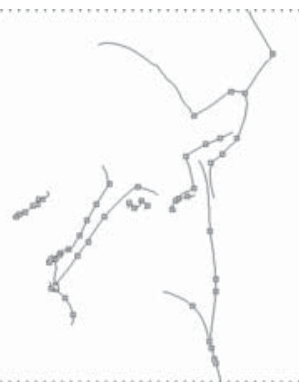

(c)

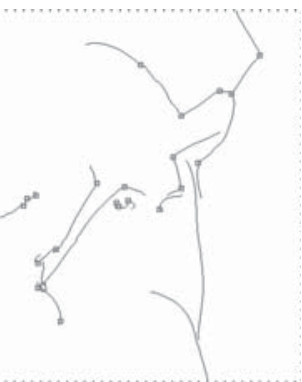

(d)

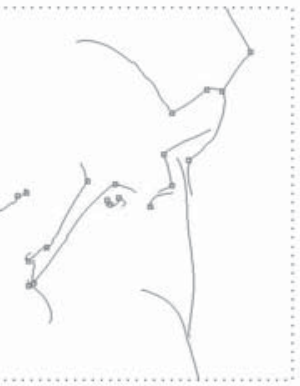

(g)

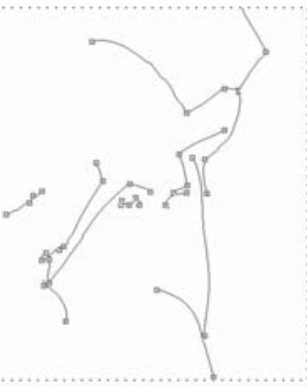

(h)

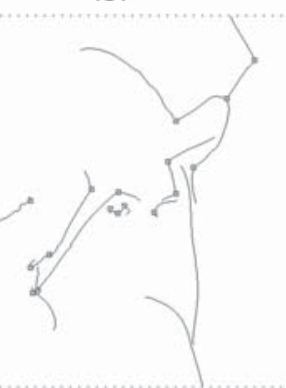

(k)

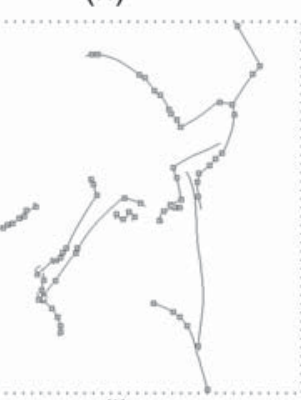

(I)

Fig. 5. Corner detection examples: (a) edge image (of original 'Lena' image) obtained using the edge extraction and selection setup in [5] and ground truth corners, (b) AD [30], (c) RJ [36], (d) CSS [27], (e) He \& Yung [18], (f) MSCP [45], (g) ARCSS [5], (h) Eigenvalue [40], (i) CPDA [6], (j) Zhang [46], (k) Fast-CPDA [7] and (l) GCM [47].

In the manual system [26], corners detected in the original images by a detector are considered as detected corners and corners identified by human subjects on the same original images are considered as ground truth. Then, ground truth and detected corners are compared to evaluate the performance of that detector. The accuracy index is the same as (2) except $N_{t}=N_{g}$, where $N_{g}$ is the number ground truth corners, $N_{r}$ is the number of corresponding corners between detected and ground truth corners. The localization error is measured using (3) where $\left(x_{t i}, y_{t i}\right)$ is replaced with the ground truth position $\left(x_{g i}, y_{g i}\right)$.

2) Data sets: The first data set had a total of 25 different original $512 \times 512$ grey-scale images including some artificial images like 'Block' and real world images like 'Lena', 'Leaf', 'House' and 
'Lab'. All of the above original images were collected from standard data sets [31], [42], [3]. The data set employed had a total of 9450 affine transformed and signal processed (test) images, which were obtained by applying the following seven approaches of operations on each original image:

$\diamond$ rotation at 18 different angles $\theta$ in $\left[-90^{\circ},+90^{\circ}\right]$ at $10^{\circ}$ apart, excluding $0^{\circ}$;

$\diamond$ uniform (U) scaling factors $s_{x}=s_{y}$ in $[0.5,2.0]$ at 0.1 apart, excluding 1.0;

$\diamond$ non-uniform (NU) scaling factors $s_{x}$ in $[0.7,1.3]$ and $s_{y}$ in $[0.5,1.8]$, at 0.1 apart, excluding the cases when $s_{x}=s_{y}$;

$\diamond$ combined transformations (rot.-scale): $\theta$ in $\left[-30^{\circ},+30^{\circ}\right]$ at $10^{\circ}$ apart, excluding $0^{\circ}$, followed by uniform or non-uniform scaling factors $s_{x}$ and $s_{y}$ in $[0.8,1.2]$ at 0.1 apart;

$\diamond$ JPEG lossy compression at 20 quality factors in $[5,100]$, at 5 apart;

$\diamond$ zero mean white Gaussian noise at 10 variances in $[0.005,0.05]$ at 0.005 apart; and

$\diamond$ shearing factors $s_{h x}$ and $s_{h y}$ in $[0,0.012]$ at 0.002 apart, excluding the one when $s_{h x}=s_{h y}=0.0$.

Therefore, the first data set had a total of 450 rotated, 375 uniform-scaled, 2925 non-uniform scaled, 3750 rotated and scaled transformed images. It also had 500 JPEG compressed, 250 Gaussian noise-induced and 1200 sheared images. Note that transformations comprising rotations were also followed by cropping that removed the outer black parts. Consequently, many detected corners in the original images were cropped off in the test images for the transformations involving rotations.

The second data set is regarded as a standard by the computer vision community [24]. ${ }^{1}$ It consists of eight sets of images, where each set has an original image and five projectively transformed images. The operations include viewpoint change ( 2 sets), scaling (zoom and rotation, 2 sets), blurring (2 sets), illumination (light) change (1 set) and JPEG compression (1 set).

3) Detectors: Eleven recently developed detectors, as shown in Table II, that have been found to yield consistent results, have been evaluated. Six of them are direct curvature estimating detectors and the other five are indirect curvature estimating detectors. All detectors were assigned default parameter settings indicated by their authors, except the RJ [36], AD [30] and Eigenvalue [40] detectors for which direct smoothing was used to enhance their performance.

In addition, the Harris-Laplace [24], SURF (Speeded Up Robust Features) [8] and LoG (Laplacian of the Gaussian) [19] interest-point detectors have been used to compare the performance of contourbased detectors with interest-point detectors. While the Harris-Laplace detector detects corners, the other two interest-point detectors detect blobs (a description of the local neighbourhood around a detected point) [41]. The Harris-Laplace was the best performing interest-point detector in similarity transformations and the LoG detector also performed close to the Harris-Laplace detector [24]. Unlike the study in [24], it was observed that the LoG detector performed slightly better than the Harris-

\footnotetext{
${ }^{1}$ Available at: http://www.featurespace.org/
} 
Laplace detector in the study presented below. The reasons were possibly due to use of different metrics for repeatability estimation and unavailability of some images in the test sequences of the second data set which were used in [24].

\section{Results and Discussions}

Fig. 5 shows ground truth and corner detection examples on the 'Lena' image. The evaluation results presented below are averaged over the whole data set. It is seen that while the detectors using indirect curvature estimation techniques [6], [46] are more robust, the detectors using direct curvature estimation techniques [45], [27] are more efficient.

1) Accuracy: Table III shows the accuracy and localization error on the first data set using the manual evaluation system [26]. While the CPDA detector offered the highest accuracy, the Zhang detector [46] had the lowest localization error. Among the detectors using indirect curvature, the CPDA, Fast-CPDA and Zhang detectors performed better than those using direct curvature. The GCM and Eigenvalue detectors performed worse than the CSS and MSCP detectors as they detected a lot of false corners, as shown in Figs. 5(h) and 5(l). Among the detectors using direct curvature, the MSCP detector performed the best and the RJ detector performed the worst, as it detected a large number of false corners as shown in Fig. 5(c).

2) Robustness: Figs. 6 and 7 present the results under geometric transformations and signal processing operations, respectively, using the first data set. Fig. 8 shows the same using the second data set.

In both data sets, the indirect curvature estimating detectors were more robust than their direct curvature estimating counterparts both in terms of average repeatability and localization error. More specifically, CPDA, Fast-CPDA, Zhang and Eigenvalue detectors mostly occupied the first four positions in the ranking. In the first data set, among these four detectors, the CPDA and Fast-CPDA detectors performed better than the Zhang and Eigenvalue detectors in rotation, scaling and noising operations. The former two showed slightly lower repeatability, but lower (better) localization error in rotation-scaling, shearing and JPEG operations (Figs. 6 and 7). In addition, in the second data set, the GCM detector performed better in viewpoint change and JPEG compression, the CPDA detector in blurring operations, and the Fast-CPDA detector in scaling and light change than the Zhang and Eigenvalue detectors. The largest localization error was produced by the AD detector (Fig. 8).

In the first data set, among the detectors using indirect curvature estimation techniques, the GCM detector performed the worst in geometric transformations (see Fig. 6). However, it offered the highest repeatability in signal processing operations but suffered from higher localization error than the CPDA and Fast-CPDA detectors (see Fig. 7). In the second data set, this detector also gave the highest repeatability in viewpoint change and JPEG compression operations, but suffered from 


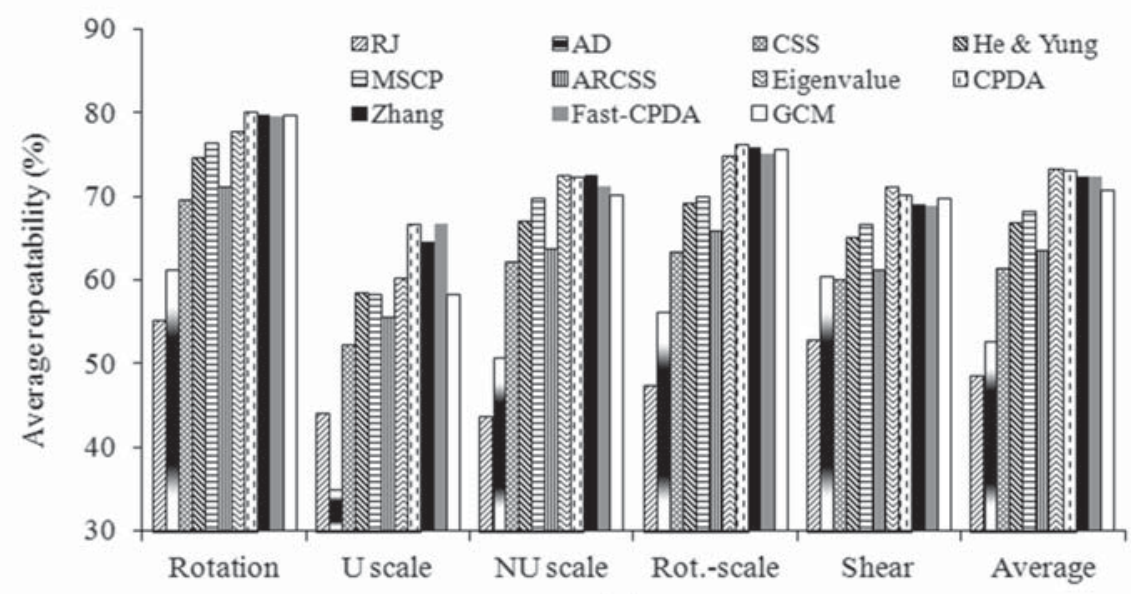

(a)

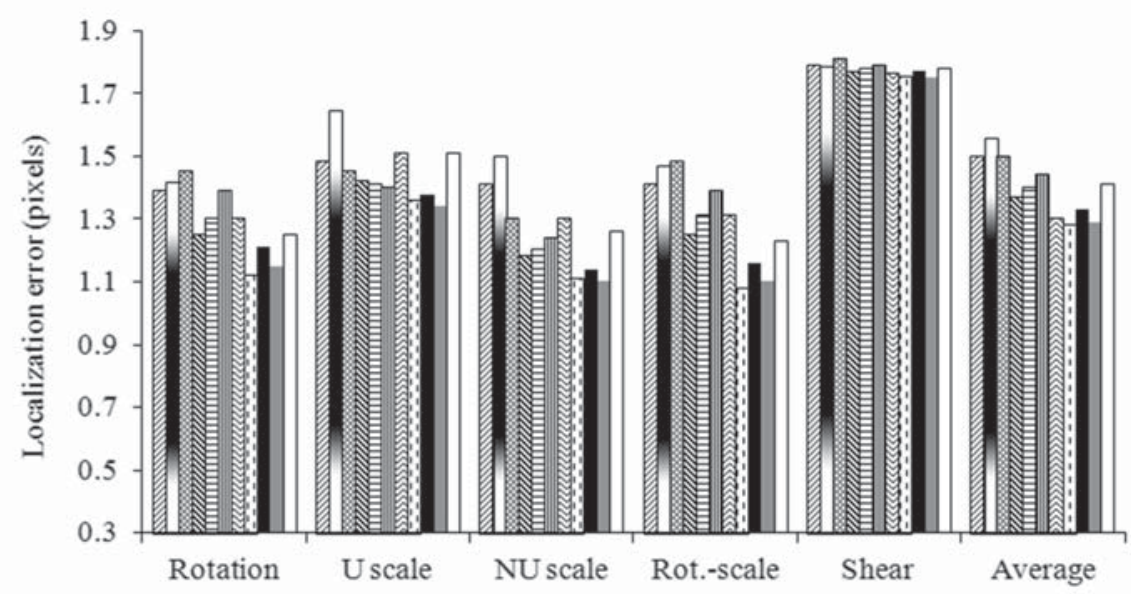

(b)

Fig. 6. Comparative results in geometric transformations (Data set 1).

higher localization error than the CPDA and Fast-CPDA detectors. There are four main reasons for its behaviour. Firstly, its significance measure (determinant of gradient correlation matrix) is highly sensitive to geometric transformations. Secondly, it uses first-order derivatives, which often yield a high significance measure within a local variation area of a curve, as shown in Fig. 3. Thirdly, it uses a very small neighbourhood $(1 \times 1)$ around each pixel during estimation of significance measure. Finally, it does not adopt any technique to remove false corners. Consequently, the GCM detector detects many false corners (see Fig. 5(1)). These false corners are repeatedly detected in the test images also. Therefore, although it showed high repeatability in signal processing operations and thereby ranked second under robustness (Tables IV and V), it ranked eighth under accuracy (Table III). 


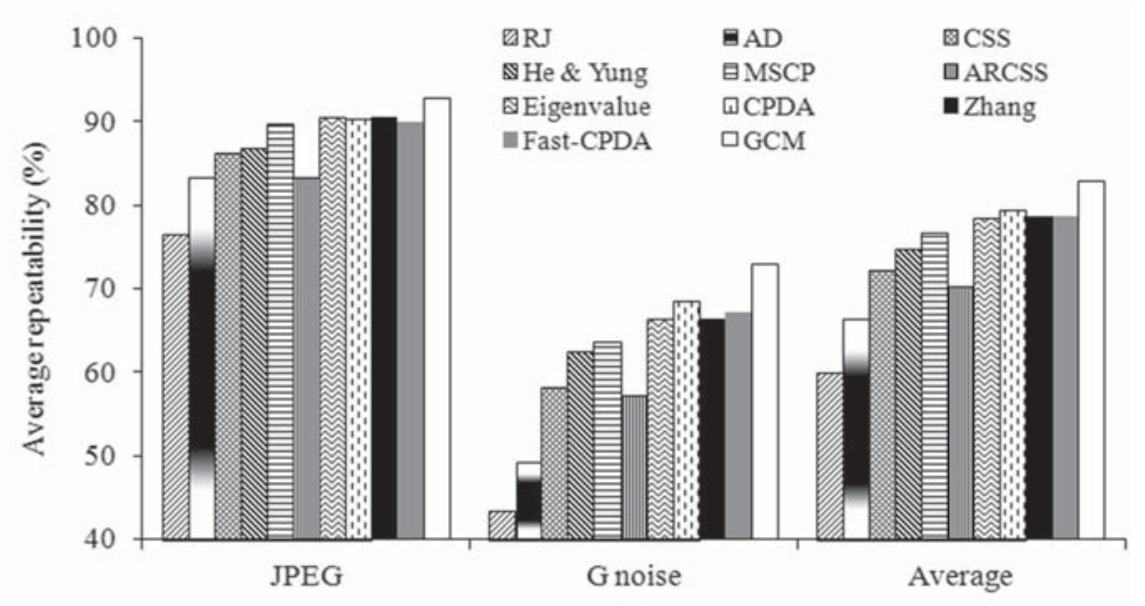

(a)

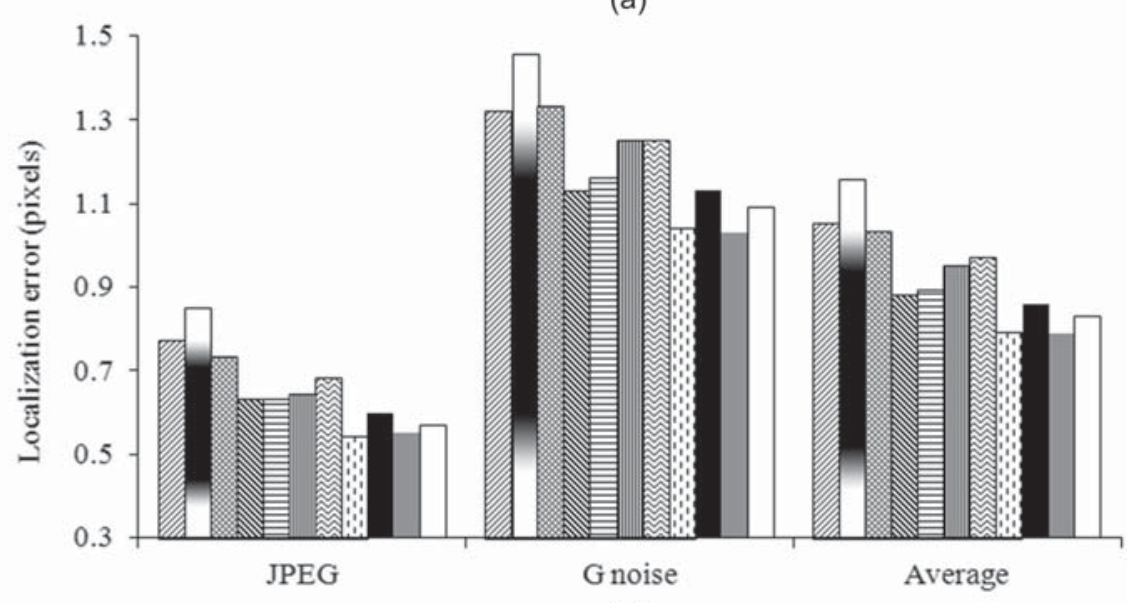

(b)

Fig. 7. Comparative results in signal processing operations (Data set 1).

In the first data set, among the detectors estimating direct curvatures, the RJ detector showed the lowest repeatability and the AD detector suffered the highest localization error in both geometric and signal processing attacks. The MSCP and He \& Yung detectors were more robust than the CSS and ARCSS detectors. The RJ detector yielded many false corners (see Fig. 5(c)), which were not stable under different operations. A similar trend was observed for the direct curvature estimating detectors in the second data set, except the AD detector performed the worst among all the detectors. The reason was, like the RJ detector, it estimated the $k$-cosine curvature and therefore detected a lot of false candidates. While trying to remove the false candidates using the anisotropic diffusion, it removed some true corners as well but failed to remove all false candidates (see Fig. 5(b)). A coarse-to-fine corner tracking step was also necessary to improve the localization of the detected corners. 


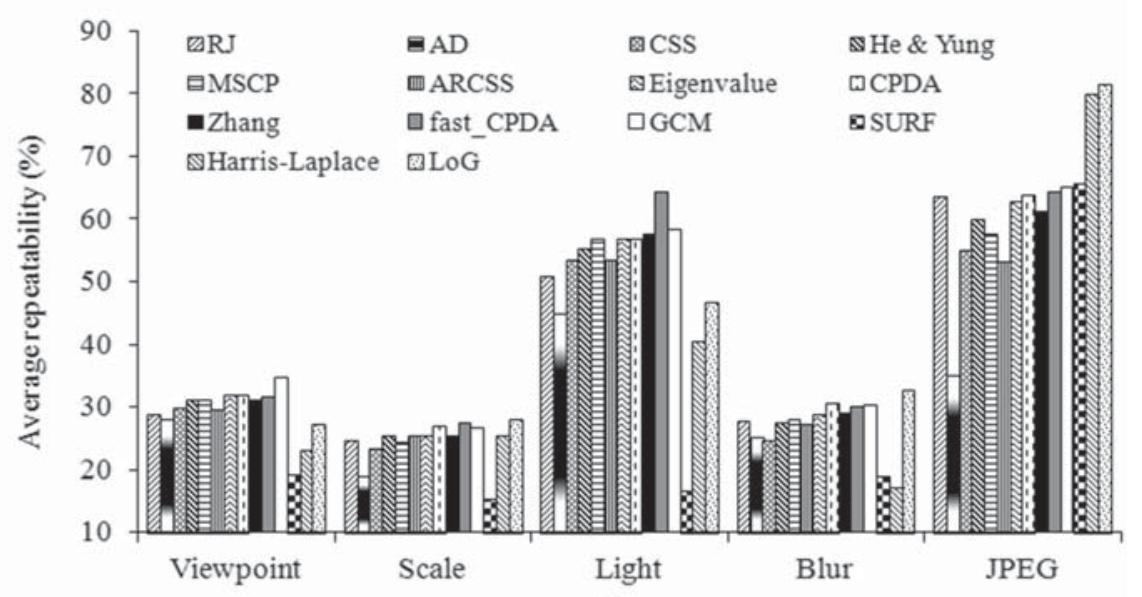

(a)

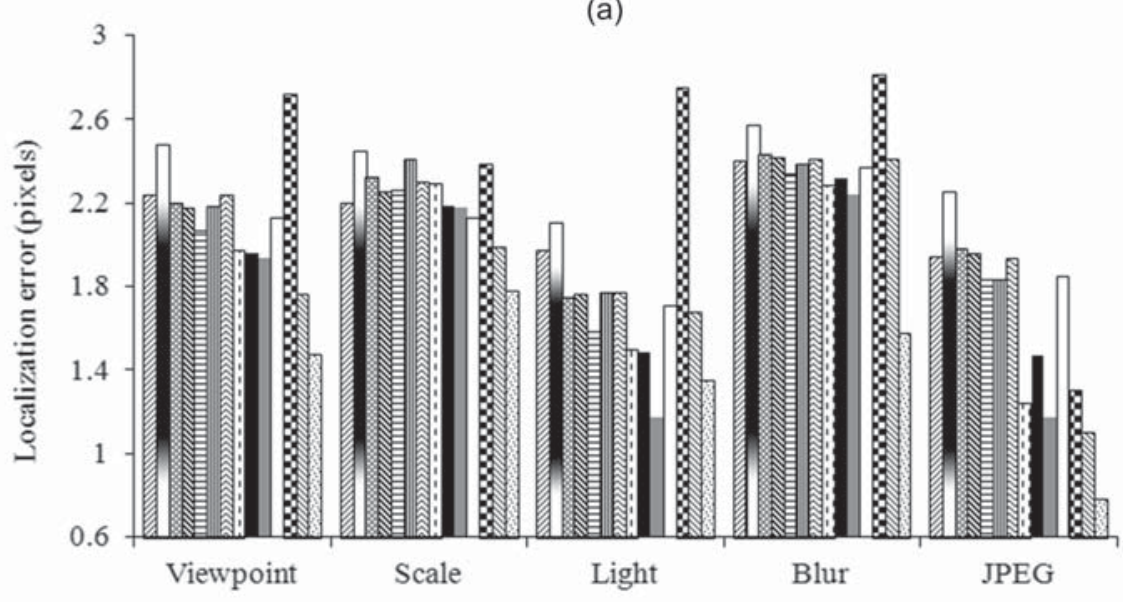

(b)

Fig. 8. Comparative results in different operations (Data set 2).

In the second data set, the Harris-Laplace interest-point detector offered lower average repeatability than the contour-based corner detectors in viewpoint change, light change and blurring. The SURF detector performed worse (lower repeatability and higher localization error) than the contour-based detectors in all geometric transformations, light change and blurring. However, all three interestpoint detectors offered significantly higher repeatability than the contour-based detectors in JPEG compression operation. Moreover, the LoG detector performed better than the contour-based corner detectors in scale change and blurring.

Fig. 9 shows the average repeatability of top six detectors (4 contour-based and 2 interest-point detectors) in different viewpoint and scale (scaled and rotated, as shown in Fig. 10) changes. The CPDA, Fast-CPDA and Eigenvalue detectors showed similar results in viewpoint change. The CPDA, 


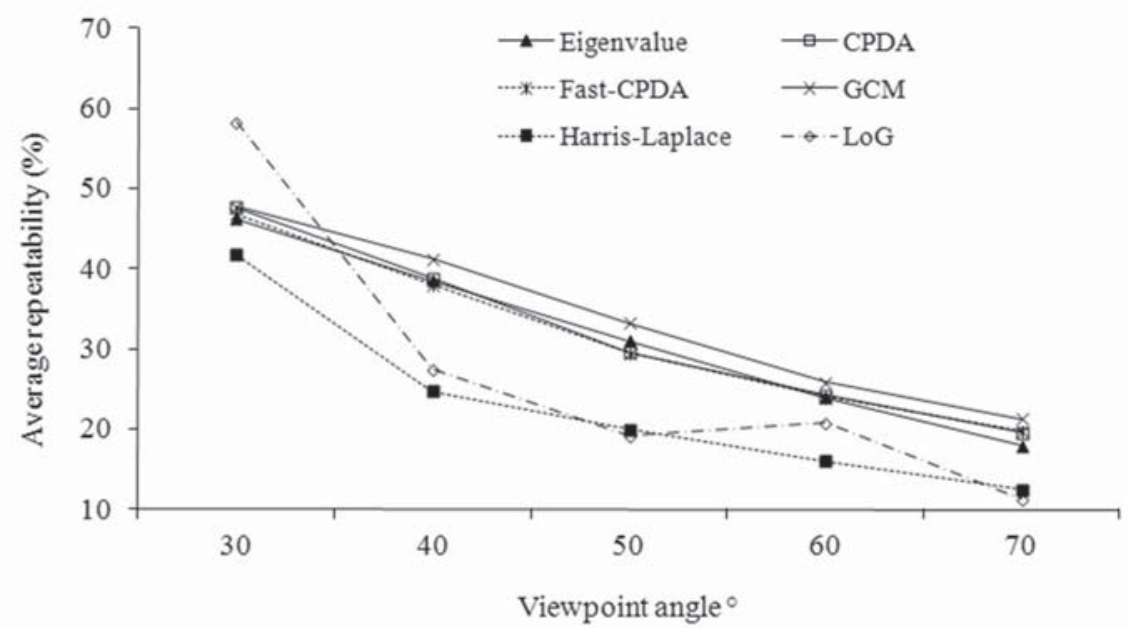

(a)

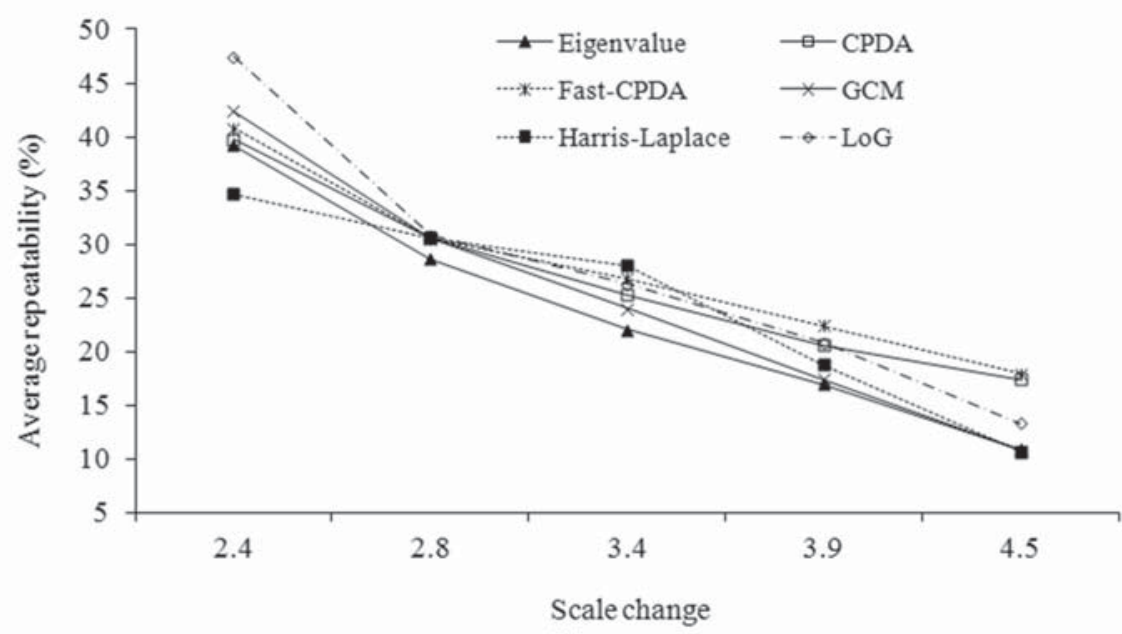

(b)

Fig. 9. Average repeatability of top six detectors in different viewpoint and scale changes.

Fast-CPDA and LoG detectors performed better in scale change than the Eigenvalue and GCM detectors. The GCM detector offered higher repeatability in viewpoint change than other detectors. However, as discussed above it detected a lot of false corners which were repeated in the test images as well. In addition, it suffered from higher localization error.

The above results are summarized in Tables IV and V. In the first data set, the CPDA detector had the highest robustness among all the detectors in terms of both average repeatability rate and localization error. The GCM and Zhang and Fast-CPDA detectors performed close to the CPDA detector. In the second data set, the Fast-CPDA detector offered the highest repeatability but the LoG detector suffered the lowest localization error. The CPDA detector performed close to the Fast-CPDA 

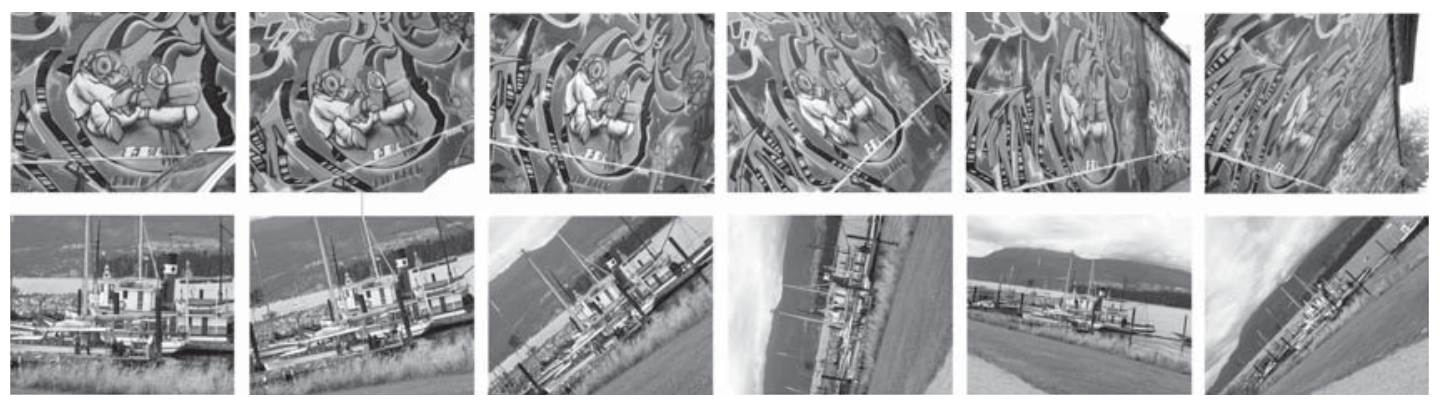

Fig. 10. Two sets of images from the second data set: graffiti (viewpoint change, top row) and boat (zoom and rotation, bottom row).

and LoG detectors. The Eigenvalue detector had the highest localization error among the detectors using indirect curvatures. Among the detectors using direct curvatures, the MSCP and $\mathrm{He} \&$ Yung detectors performed better than the CSS and ARCSS detectors.

Moreover, it was observed that the contour-based detectors are less robust to projective transformations (the second data set, Table V) than to affine transformations (the first data set, Table IV). The reason is that while in affine transformations straight lines remain straight, parallel lines remain parallel, and rectangles might become parallelograms; in projective transformations straight lines remain straight but parallel lines converge toward vanishing points which can fall inside or outside the image, even at infinity. Moreover, the transformations performed on the second data set produced more significant geometric perturbations than those in the first data set. Fig. 10 shows two sets of images from the second data set.

Note that in the reported performance comparisons, average repeatability and localization error were estimated from many thousand corner detections. Many of these detected corners were quite stable, i.e., they could be detected by most detectors. However, there were difficult cases where some corners were missed or weak or false corners were detected. The CPDA, Fast-CPDA, and Zhang detectors performed better than others in such difficult situations. Therefore, though the average repeatability and localization error of these detectors seem to be very close to each other, their performance difference is quite significant.

3) Efficiency: Table VI compares the running time of different detectors on a Windows XP machine with $3.00 \mathrm{GHz}$ of Intel(R) Core(TM)2 Duo CPU and 3.23GB of RAM (using the first data set).

The Fast-CPDA is the fastest detector followed by the MSCP and CSS detectors. The Fast-CPDA detector estimates curvature only on selected limited number of curve-points. The MSCP detector simply applies a curvature-threshold to the curvature product function and all three detectors do not follow any corner tracking step. The CSS detector estimates curvature on a single medium scale 
( $\sigma=4)$ and tracks the detected corners to $\sigma=1$. On the other hand, the CPDA detector consumes a lot of time in curvature estimation using three chords. He \& Yung, though a single-scale detector, was slow because it spends much time in adaptive curvature-threshold calculation using dynamic RoS. Eigenvalue detector is a slower detector, since it estimates the eigenvalues of covariance metrics using a $10 \times 10$ neighbourhood around each pixel. The $\mathrm{AD}$ detector is the slowest detector since it iteratively smoothes the curvature function using the anisotropic diffusion technique.

From Table VI it is also evident that, except for the Fast-CPDA and AD detectors, the detectors estimating direct curvatures are faster than those estimating indirect curvatures. Since the running time of detectors can be insignificant with even more powerful computers, it can be said that CPDA, Fast-CPDA and Zhang detectors perform the best.

\section{CONCLUSION}

In this paper, the general framework of contour-based detectors has been presented. There are five main detection steps: edge extraction and selection, curve smoothing, curvature estimation, finding corners and coarse-to-fine corner tracking. The 'corner tracking' step is an optional step. The 'curve smoothing' and 'curvature estimation' steps are sometimes integrated, for example, for detectors estimating indirect curvature with an indirect smoothing technique.

Out of five steps, curve smoothing and curvature estimation are the most crucial steps as they significantly control the detection performance. There are two types of smoothing: direct and indirect. The curvature estimation techniques are also categorized into two groups: direct and indirect. A corner detector can use direct smoothing, indirect smoothing or both, but it estimates curvature using only direct or indirect techniques. As the detectors estimating indirect curvatures use a large neighbourhood, their curvature estimations are more stable than their direct counterparts.

A comparative study of selected contour-based detectors has been carried out using both automatic (robustness) and manual evaluation (accuracy) systems. Experientially, it has been observed that while the detectors using indirect curvature estimation techniques are more accurate and robust, the detectors using direct curvature estimation techniques are more efficient. The Harris-Laplace and LoG detectors, which are well regarded interest-point detectors, though offer lower localization error, are less robust than the contour-based detectors.

\section{REFERENCES}

[1] N. Ansari and K. W. Huang. Non-parametric dominant point detection. Pattern Recognition, 24(9):849-862, Sep 1991.

[2] H. Asada and M. Brady. The curvature primal sketch. IEEE Trans. on Pattern Analysis and Machine Intelligence, 8(1):2-14, Jan 1986.

[3] M. Awrangjeb. Image database and corner detection, 2010, http://personal.gscit.monash.edu.au/ awran/images.html. 
[4] M. Awrangjeb. Contour-based corner detection and robust geometric point matching techniques. PhD Thesis, Monash University, Oct. 2008.

[5] M. Awrangjeb and G. Lu. An improved curvature scale-space corner detector and a robust corner matching technique for transformed image identification. IEEE Trans. Image Process., 17(12):2425-2441, Dec 2008.

[6] M. Awrangjeb and G. Lu. Robust image corner detection based on the chord-to-point distance accumulation technique. IEEE Trans. Multimedia, 10(6):1059-1072, Oct 2008.

[7] M. Awrangjeb, G. Lu, C. S. Fraser, and M. Ravanbakhsh. A fast corner detector based on the chord-to-point distance accumulation technique. In Proc. Digital Image Computing: Techniques and Applications, pages 519-525, Melbourne, Australia, Dec 2009.

[8] H. Bay, A. Ess, T. Tuytelaars, and L. Van Gool. SURF: Speeded Up Robust Features. Computer Vision and Image Understanding, 110(3):346-359, 2008.

[9] V. Beau and M. Singer. Reduced resolution and scale space for dominant feature detection in contours. Pattern Recognition, 34(2):287-297, Feb 2001.

[10] A. Carmona-Poyato, N. L. Fernndez-Garca, R. Medina-Carnicer, and F. J. Madrid-Cuevas. Dominant point detection: A new proposal. Image and Vision Computing, 23(13):1226-1236, Nov 2010.

[11] A. Carmona-Poyato, F. J. Madrid-Cuevas, R. Medina-Carnicer, and R. Muoz-Salinas. Polygonal approximation of digital planar curves through break point suppression. Pattern Recognition, 43(1):14-25, 2010.

[12] A. Carmona-Poyato, R. Medina-Carnicer, F. J. Madrid-Cuevas, R. Muoz-Salinas, and N. L. Fernndez-Garca. A new measurement for assessing polygonal approximation of curves. Pattern Recognition, 44(1):45-54, Jan 2011.

[13] M. A. Fischler and R. C. Bolles. Perceptual organization and curve partitioning. IEEE Trans. on Pattern Analysis and Machine Intelligence, 8(1):100-105, Jan 1986.

[14] H. Freeman. On the encoding of arbitrary geometric configurations. IRE Trans. on Electronic Computers, EC10(2):260-268, Jun 1961

[15] X. Gao, F. Sattar, A. Quddus, and R. Venkateswarlu. Multiscale contour corner detection based on local natural scale and wavelet transform. Image and Vision Computing, 25(6):890-898, Jun 2007.

[16] X. Gao, W. Zhang, F. Sattar, R. Venkateswarlu, and E. Sung. Scale-space based corner detection of gray level images using plessey operator. In Proc. International Conference on Information, Communications and Signal Processing, Bangkok, Thailand, Dec. 2005.

[17] D. S. Guru, R. Dinesh, and P. Nagabhushan. Boundary based corner detection and localization using new 'cornerity' index: a robust approach. In Proc. Canadian Conference on Computer and Robot Vision, Ontario, Canada, May 2004.

[18] X. C. He and N. H. C. Yung. Corner detector based on global and local curvature properties. Optical Engineering, 47(5):057008-1-057008-12, May 2008.

[19] T. Lindeberg. Feature detection with automatic scale election. International Journal of Computer Vision, 30(2):79-116, 1998.

[20] M. Marji and P. Siy. Polygonal representation of digital planar curves through dominant point detection - a nonparametric algorithm. Pattern Recognition, 37(11):2113-2130, Nov 2004.

[21] A. Masood and M. Sarfraz. Corner detection by sliding rectangles along planar curves. Computers \& Graphics, 31(3):440-448, Jun 2007.

[22] A. Masood. Optimized polygonal approximation by dominant point deletion. Pattern Recognition, 41(1):227-239, 2008.

[23] K. Mikolajczyk and C. Schmid. Indexing based on scale invariant interest points. In Proc. Int. Conf. on Computer Vision, pages 525-531, Vancouver, Canada, July 2001.

[24] K. Mikolajczyk and C. Schmid, "Scale \& affine invariant interest point detectors", International Journal of Computer Vision, 60(1):63-86, Oct 2004. 
[25] F. Mokhtarian and F. Mohanna. Enhancing the curvature scale space corner detector. In Proc. Scandinavian Conference on Image Analysis, pages 145-152, 2001.

[26] F. Mokhtarian and F. Mohanna. Performance evaluation of corner detectors using consistency and accuracy measures. Computer Vision and Image Understanding, 102(1):81-94, Apr. 2006.

[27] F. Mokhtarian and R. Suomela. Robust image corner detection through curvature scale space. IEEE Trans. Pattern Anal. Mach. Intell., 20(12):1376-1381, Dec. 1998.

[28] T. P. Nguyen and I. Debled-Rennesson. A discrete geometry approach for dominant point detection. Pattern Recognition., 44(1):32-44, 2011.

[29] M. T. Parvez and S. A. Mahmoud. Polygonal approximation of digital planar curves through adaptive optimizations. Pattern Recognition Letters., 31(13):1997-2005, 2010.

[30] G. V. Pedrosa and C. A. Z. Barcelos. Anisotropic diffusion for effective shape corner point detection. Pattern Recognition Letters., 31(12):1658-1664, Sep. 2010.

[31] F. A. P. Petitcolas. Photo database, 2010, http://www.petitcolas.net/fabien/watermarking/image_database/index.html.

[32] A. M. G. Pinheiro and M. Ghanbari. Piecewise approximation of contours through scale-space selection of dominant points. IEEE Trans. Image Process., 19(6):1442-1450, Jun. 2010.

[33] U. Ramer. An iterative procedure for the polygonal approximation of plane curves. Computer Graphics and Image Processing, 1(3):244-256, Nov 1972.

[34] A. Rattarangsi and R. T. Chin. Scale-based detection of corners of planar curves. IEEE Trans. Pattern Anal. Mach. Intell., 14(4):430-449, Apr. 1992.

[35] B. K. Ray and R. Pandyan. ACORD-an adaptive corner detector for planar curves. Pattern Recognistion, 36(2003):703$708,2003$.

[36] A. Rosenfeld and E. Johnston. Angle detection on digital planar curves. IEEE Trans. Comput., C-22:875-878, Sep 1973.

[37] A. Rosenfeld and J. Weszka. An improved method of angle detection on digital curves. IEEE Trans. Comput., C-24:940-941, Sep 1975.

[38] P. L. Rosin. Determining local natural scales of curves. Pattern Recognition Letters, 19(1):63-75, May 1998.

[39] C. H. Teh and R. T. Chin. On the detection of dominant points on digital curves. IEEE Trans. on Pattern Analysis and Machine Intelligence, 11(8):859-872, Aug 1989.

[40] D.-M. Tsai, H. T. Hou, and H. J. Su. Boundary-based corner detection using eigenvalues of covariance matrices. Pattern Recognition Letters, 20(1):31-40, Jan 1999.

[41] T. Tuytelaars and K. Mikolajczyk. Local invariant feature detectors - a survey. Computer Graphics and Visison, 3(3):177-280, 2007.

[42] USC-SIPI. The usc-sipi image database, 2010, http://sipi.usc.edu/database/.

[43] W. Y. Wu. Dominant point detection using adaptive bending value. Image and Vision Computing, 21(6):517-525, Jun 2003.

[44] C. H. Yeh. Wavelet-based corner detection using eigenvectors of covariance matrices. Pattern Recognition Letters, 24(15):2797-2806, Nov 2003.

[45] X. Zhang, M. Lei, D. Yang, Y. Wang, and L. Ma. Multi-scale curvature product for robust image corner detection in curvature scale space. Pattern Recognistion Letters, 28(2007):545-554, 2007.

[46] X. Zhang, H. Wang, M. Hong, L. Xu, D. Yang, and B. C. Lovell. Robust image corner detection based on scale evolution difference of planar curves. Pattern Recognition Letters, 30(1):449-455, Mar 2009.

[47] X. Zhang, H. Wang, A. W. B. Smith, X. Ling, B. C. Lovell, and D. Yang. Corner detection based on gradient correlation matrices of planar curves. Pattern Recognition, 43(4):1207-1223, Apr 2010. 
[48] X. Zhang and D. Zhao. A parallel algorithm for detecting dominant points on multiple digital curves. Pattern Recognition, 30(2):239-244, Feb 1997.

[49] P. Zhu and P. M. Chirlian. On critical point detection of digital shapes. IEEE Trans. on Pattern Analysis and Machine Intelligence, 17(8):737-748, Aug 1995.

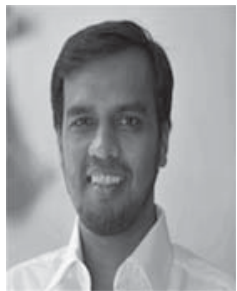

Dr. Mohammad Awrangjeb is currently a Research Fellow at the Cooperative Research Centre for Spatial Information in the Dept. of Infrastructure Engineering of the University of Melbourne. He obtained his Ph.D. in 2009 from Monash University, Australia. He worked as a lecturer in University of Asia-Pacific, Dhaka in 2002; as a Research Scholar in the National University of Singapore in 200204; and as a lecturer in American International University-Bangladesh in 2004-05. He is a recipient of Discovery Early Career Researcher Award by the Australian Research Council for the period 2012-

15. Dr. Awrangjeb's research interest includes automatic feature extraction and matching, multimedia security and image processing. He is a coauthor of more than 30 research articles in internationally renowned journals and conferences.

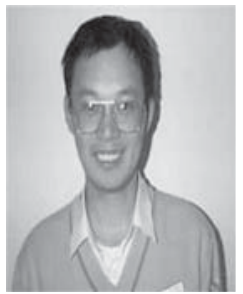

Prof. Guojun Lu is currently a Professor at Gippsland School of Information Technology, Monash University. He has held positions at Loughborough University, National University of Singapore, and Deakin University, after he obtained his PhD in 1990 from Loughborough University and BEng in 1984 from Nanjing Institute of Technology (now South East University). Guojun's main research interests are in multimedia communications and multimedia information indexing and retrieval. $\mathrm{He}$ has published over 120 refereed journal and conference papers in these areas and wrote two books Communication and Computing for Distributed Multimedia Systems (Artech House 1996), and Multimedia Database Management Systems (Artech House 1999).

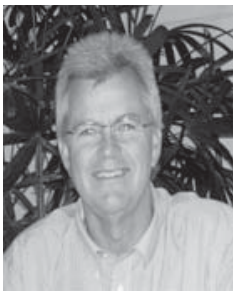

Prof. Clive S. Fraser is currently a Program Science Director in the Cooperative Research Centre for Spatial Information, and a Professorial Fellow in the Dept. of Infrastructure Engineering at the University of Melbourne. Prof. Frasers particular areas of research interest lie in digital close-range photogrammetry, including 3D forensic analysis and accident reconstruction, industrial measurement systems, and the metric exploitation of high-resolution satellite imagery. In recognition of his academic and professional work he has earned numerous international awards and has authored more than 300 scientific publications. 
TABLE I

COMPARISONS OF EVALUATION SYSTEMS AND TEST RESULTS FOR SELECTED CONTOUR-BASED DETECTORS (TYPE: M = MANUAL EVALUATION SYSTEM INVOLVING HUMAN JUDGEMENT [26], A = AUTOMATIC EVALUATION SYSTEM [6]; ERROR: MAXIMUM DISTANCE IN PIXELS BETWEEN REFERENCE AND DETECTED CORNERS FOR A REPEATED CORNER, NA = NOT APPLICABLE; DATA SET: NUMBER OF ORIGINAL AND TEST IMAGES; OPERATIONS: HOW TEST IMAGES WERE GENERATED, US $=$ UNIFORM SCALING, NUS $=$ NON-UNIFORM SCALING; TEST RESULTS $: C C N=$ CONSISTENCY OF CORNER NUMBERs [26], $A c u=$ ACCURACy [26], $T D R=$ TRUE DETECTION RATE [45], $F D R=$ FALSE DETECTION RATE [45], $L_{e}=$ LOCALIZATION ERROR [6], $E I=$ ERROR INDEX [46], $R_{a v g}=$ AVERAGE REPEATABILITy [6], Time IN SECONDS).

\begin{tabular}{|c|c|c|c|c|c|c|c|c|c|c|}
\hline \multirow{2}{*}{ References } & \multirow{2}{*}{ Type } & \multirow{2}{*}{ Error } & \multicolumn{2}{|c|}{ Data set } & \multicolumn{2}{|c|}{ Operations } & \multirow{2}{*}{ Detectors } & \multirow{2}{*}{\multicolumn{3}{|c|}{ Test results }} \\
\hline & & & Ori. & Test & Geometric & Signal & & & & \\
\hline \multirow{4}{*}{ Mokhtarian 2006} & \multirow{4}{*}{ M } & \multirow{4}{*}{3} & \multirow{4}{*}{5} & \multirow{4}{*}{590} & Rotation(90) & & \multirow{4}{*}{$\begin{array}{r}\text { CSS } \\
\text { ECSS }\end{array}$} & $C C N$ & Acu & \\
\hline & & & & & US(50) & & & 51 & 72 & \\
\hline & & & & & NUS(150) & & & 60 & 78 & \\
\hline & & & & & Affine(300) & & & & & \\
\hline \multirow{4}{*}{ Zhang 2007} & \multirow{4}{*}{ M } & \multirow{4}{*}{3} & \multirow{4}{*}{5} & \multirow{4}{*}{1365} & Rotation(90) & & \multirow{4}{*}{$\begin{array}{r}\text { CSS } \\
\text { He \& Yung } \\
\text { MSCP }\end{array}$} & $C C N$ & $A c u$ & \\
\hline & & & & & US(75) & & & 47 & 75 & \\
\hline & & & & & NUS(400) & & & 59 & 77 & \\
\hline & & & & & Affine(800) & & & 73 & 84 & \\
\hline \multirow{4}{*}{ He 2008} & \multirow{4}{*}{ M } & & & & & & & $T D R$ & $F D R$ & $L_{e}$ \\
\hline & & t & ? & & & & CSS & 93 & 21 & 1.53 \\
\hline & & 4 & 2 & - & & & ECSS & 92 & 14 & 1.33 \\
\hline & & & & & & & He \& Yung & 93 & 4 & 0.99 \\
\hline & & & & & Rotation(414) & JPEG(460) & & $R_{\text {avg }}$ & $L_{e}$ & \\
\hline & & & & & US(345) & Noise(230) & CSS & 64 & 1.36 & \\
\hline Awrangjeb 2008a & A & 3 & 23 & 8694 & NUS(2691) & & ECSS & 58 & 1.38 & \\
\hline & & & & & Affine(3450) & & ARCSS & 66 & 1.30 & \\
\hline & & & & & Shear(1104) & & & & & \\
\hline & & & & & Rotation(414) & JPEG(460) & & $R_{\text {avg }}$ & $L_{e}$ & \\
\hline & & & & & US(345) & Noise(230) & RJ & 52 & 1.37 & \\
\hline & & & & & NUS(2691) & & CSS & 65 & 1.36 & \\
\hline Awrangjeb 2008b & A & 3 & 23 & 8694 & Affine(3450) & & ARCSS & 66 & 1.30 & \\
\hline & & & & & Shear(1104) & & He \& Yung & 70 & 1.23 & \\
\hline & & & & & & & MSCP & 71 & 1.26 & \\
\hline & & & & & & & CPDA & 73 & 1.18 & \\
\hline & & & & & Rotation(320) & Noise(200) & & $A c u$ & $E I$ & \\
\hline Zhang 2009 & 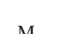 & Na & 20 & 7320 & US(200) & & CSS & 75 & 40 & \\
\hline Eicang 2005 & & & & & NUS(2200) & & Zhang & 78 & 33 & \\
\hline & & & & & Affine(4400) & & & & & \\
\hline & & & & & Rotation(414) & JPEG(460) & & $R_{\text {avg }}$ & $L_{e}$ & Time \\
\hline & & & & & US(345) & Noise(230) & CSS & 64 & 1.36 & 0.008 \\
\hline & & & & & NUS(2691) & & ARCSS & 65 & 1.30 & 0.016 \\
\hline Awrangjeb 2009 & A & 3 & 23 & 8694 & Affine(3450) & & He \& Yung & 69 & 1.23 & 0.009 \\
\hline & & & & & Shear(1104) & & MSCP & 70 & 1.25 & 0.006 \\
\hline & & & & & & & CPDA & 75 & 1.14 & 0.013 \\
\hline & & & & & & & Fast-CPDA & 74 & 1.15 & 0.005 \\
\hline & & & & & Rotation(320) & Noise(200) & & $A c u$ & $L_{e}$ & Time \\
\hline & & & & & US(300) & & GCM & 72 & 1.46 & 1.075 \\
\hline & & & & & $\operatorname{NUS}(600)$ & & CSS & 66 & 1.48 & 1.144 \\
\hline & & & & & Affine(520) & & CPDA & 67 & 1.52 & 1.219 \\
\hline Zhang 2010 & M & 3 & 20 & 1940 & & & He \& Yung & 65 & 1.45 & 1.051 \\
\hline & & & & & & & MSCP & 70 & 1.45 & 1.138 \\
\hline & & & & & & & Eigenvalue & 66 & 1.53 & 1.502 \\
\hline & & & & & & & Eigenvector & 56 & 1.70 & 1.570 \\
\hline & & & & & & & Wavelet & 60 & 1.80 & 1.139 \\
\hline
\end{tabular}


TABLE II

DETECTORS IN THE COMPARATIVE STUdy ( $L_{c}$ : CURVE-LENGTH).

\begin{tabular}{l|l|l|l}
\hline \hline \multirow{2}{*}{ Detectors (References) } & \multicolumn{2}{|c|}{ Smoothing } & \multirow{2}{*}{ Curvature } \\
\cline { 2 - 3 } & Direct $\sigma, \rho$ & Indirect $k, L$ & \\
\hline RJ [36] & $\sigma=3$ & $k=0.1 \times L_{c}$ & Direct \\
CSS [27] & $\sigma=4$ & - & Direct \\
He \& Yung [18] & $\sigma=3$ & $k$ : dynamic & Direct \\
MSCP [45] & $\sigma=3,3.5,4$ & - & Direct \\
ARCSS [5] & $\sigma=3,4,5$ & - & Direct \\
AD [30] & $\sigma=1, \rho=1$ & $k=1$ & Direct \\
Eigenvalue [40] & $\sigma=3$ & $k=10$ & Indirect \\
CPDA [6] & $\sigma=1,2,3$ & $L=10,20,30$ & Indirect \\
Zhang [46] & $\sigma=2.5,3.75$ & - & Indirect \\
Fast-CPDA [7] & $\sigma=3,4$ & $L=10,20,30$ & Indirect \\
GCM [47] & $\sigma=3$ & $k=1$ & Indirect \\
\hline \hline
\end{tabular}

TABLE III

RANKING OF DETECTORS UNDER ACCURACY [26] USING THE FIRST DATA SET.

\begin{tabular}{c|l|l|l|l}
\hline \multirow{2}{*}{ Ranks } & \multicolumn{2}{|c|}{ Accuracy } & \multicolumn{2}{c}{ Localization error } \\
\cline { 2 - 5 } & Detectors & percentage & Detectors & pixels \\
\hline 1 & CPDA* & 91.35 & Zhan** & 1.13 \\
2 & Fast-CPDA* & 90.68 & MSCP & 1.14 \\
3 & MSCP & 88.45 & CPDA* & 1.16 \\
4 & Zhang* & 87.54 & He \& Yung & 1.24 \\
5 & CSS & 83.58 & ARCSS & 1.25 \\
6 & Eigenvalue* & 82.97 & CSS & 1.26 \\
7 & He \& Yung & 75.28 & Fast-CPDA* & 1.28 \\
8 & GCM* & 73.23 & GCM* & 1.33 \\
9 & ARCSS & 70.46 & Eigenvalue* & 1.50 \\
10 & AD & 48.47 & AD & 2.05 \\
11 & RJ & 47.08 & RJ & 2.06 \\
\hline \hline
\end{tabular}

* indicates detectors using indirect curvatures. 
TABLE IV

RANKING OF DETECTORS UNDER ROBUSTNESS [6] USING THE FIRST DATA SET.

\begin{tabular}{c|l|l|l|l}
\hline \hline \multirow{2}{*}{ Ranks } & \multicolumn{2}{|c|}{ Average repeatability } & \multicolumn{2}{c}{ Localization error } \\
\cline { 2 - 5 } & Detectors & percentage & Detectors & pixels \\
\hline 1 & CPDA* & 74.74 & CPDA* & 1.14 \\
2 & GCM* & 74.18 & Fast-CPDA* & 1.15 \\
3 & Zhang* & 74.14 & Zhang* & 1.20 \\
4 & Fast-CPDA* & 74.10 & He \& Yung & 1.23 \\
5 & Eigenvalue* & 73.21 & GCM* & 1.24 \\
6 & MSCP & 70.49 & MSCP & 1.26 \\
7 & He \& Yung & 68.97 & ARCSS & 1.30 \\
8 & ARCSS & 65.36 & Eigenvalue* & 1.30 \\
9 & CSS & 64.44 & CSS & 1.36 \\
10 & AD & 56.60 & RJ & 1.37 \\
11 & RJ & 51.76 & AD & 1.45 \\
\hline \hline
\end{tabular}

* indicates detectors using indirect curvatures.

TABLE V

RANKING OF DETECTORS UNDER ROBUSTNESS [6] USING THE SECOND DATA SET.

\begin{tabular}{|c|c|c|c|c|}
\hline \multirow{2}{*}{ Ranks } & \multicolumn{2}{|c|}{ Average repeatability } & \multicolumn{2}{|c|}{ Localization error } \\
\hline & Detectors & percentage & Detectors & pixels \\
\hline 1 & Fast-CPDA* & 38.33 & $\mathrm{LoG}^{\dagger}$ & 1.47 \\
\hline 2 & $\mathrm{GCM}^{*}$ & 38.24 & Fast-CPDA* & 1.88 \\
\hline 3 & $\mathrm{LoG}^{\dagger}$ & 37.81 & Harris-Laplace $^{\dagger}$ & 1.88 \\
\hline 4 & CPDA* & 37.33 & CPDA* & 1.98 \\
\hline 5 & Eigenvalue* & 36.33 & Zhang* & 1.98 \\
\hline 6 & Zhang* & 36.30 & MSCP & 2.09 \\
\hline 7 & He \& Yung & 35.33 & $\mathrm{GCM}^{*}$ & 2.10 \\
\hline 8 & MSCP & 34.98 & He \& Yung & 2.17 \\
\hline 9 & RJ & 34.49 & ARCSS & 2.19 \\
\hline 10 & CSS & 32.84 & RJ & 2.19 \\
\hline 11 & Harris-Laplace $^{\dagger}$ & 31.29 & Eigenvalue* & 2.20 \\
\hline 12 & $\mathrm{AD}$ & 28.05 & CSS & 2.20 \\
\hline 13 & ARCSS & 27.01 & $\mathrm{AD}$ & 2.42 \\
\hline 14 & $\mathrm{SURF}^{\dagger}$ & 23.52 & $\mathrm{SURF}^{\dagger}$ & 2.48 \\
\hline
\end{tabular}


TABLE VI

RANKING OF DETECTORS USING RUNNING TIME (PER IMAGE).

\begin{tabular}{c|l|l}
\hline \hline \multirow{2}{*}{ Ranks } & \multicolumn{2}{|c}{ Running time } \\
\cline { 2 - 3 } & Detectors & seconds \\
\hline 1 & Fast-CPDA* & 0.0048 \\
2 & MSCP & 0.0056 \\
3 & CSS & 0.0084 \\
4 & He \& Yung & 0.0090 \\
5 & CPDA* & 0.0131 \\
6 & Zhang* & 0.0146 \\
7 & ARCSS & 0.0156 \\
8 & GCM* & 0.0252 \\
9 & RJ & 0.0253 \\
10 & Eigenvalue* & 0.0444 \\
11 & AD & 0.1745 \\
\hline \hline
\end{tabular}

* indicates detectors using indirect curvatures. 